\title{
EXTREMAL SOLUTIONS AND STRONG RELAXATION FOR NONLINEAR PERIODIC EVOLUTION INCLUSIONS
}

\author{
NIKOLAOS S. PAPAGEORGIOU AND NIKOLAOS YANNAKAKIS \\ National Technical University, Department of Mathematics, Zografou Campus, \\ Athens 157 80, Greece (npapg@math.ntua.gr)
}

(Received 12 November 1998)

\begin{abstract}
We study the existence of extremal periodic solutions for nonlinear evolution inclusions defined on an evolution triple of spaces and with the nonlinear operator $A$ being time-dependent and pseudomonotone. Using techniques of multivalued analysis and a surjectivity result for $L$-generalized pseudomonotone operators, we prove the existence of extremal periodic solutions. Subsequently, by assuming that $A(t, \cdot)$ is monotone, we prove a strong relaxation theorem for the periodic problem. Two examples of nonlinear distributed parameter systems illustrate the applicability of our results.
\end{abstract}

Keywords: periodic solution; evolution triple; compact embedding; monotone operator; pseudomonotone operator; $L$-generalized pseudomonotone operator

AMS 1991 Mathematics subject classification: Primary 34G20; 35K55; 35R70

\section{Introduction}

Periodic evolution equations have been studied in the past using a variety of hypotheses and methods. We can get an idea of these methods by looking at the works of Browder [4], Pruss [19] and Becker [3]. Browder imposed monotonicity conditions on the nonlinear perturbation term, Pruss used a Nagumo-type tangential condition, and Becker has a Carathéodory nonlinear perturbation term, but assumed that the unbounded linear term (modelling the partial differential operator) is strictly $m$-accretive.

The same problem was recently studied in the context of evolution inclusions. Evolution inclusions are evolution equations with multivalued terms. Most of the works on evolution inclusions deal with the Cauchy problem. We refer to the works of Ahmed [1], Migorski [12], Papageorgiou [13,14], Papageorgiou and Shahzad [18], and references therein. More general boundary-value problems but for semilinear inclusions were examined by Anichini and Zecca [2], Papageorgiou [15], and Ding and Kartsatos [5]. The only works dealing with the nonlinear periodic problem are those by Vrabie [21], Hirano [6], Hu and Papageorgiou [8] and Papageorgiou et al. [17]. Vrabie extends the aforementioned work of Becker and assumes a multivalued strongly $m$-accretive and time-independent nonlinear operator $A$ and a single-valued Carathéodory function $f(t, x)$ on the right-hand side. Hirano deals with a subdifferential evolution inclusion with a Carathéodory righthand side and drops the strong monotonicity hypothesis, replacing it with a unilateral 
condition. Hu and Papageorgiou extend the work of Pruss to a nonlinear, set-valued setting and assume a time-varying, maximal monotone nonlinear operator $A$ and a setvalued right-hand side term $F(t, x)$, which has closed and convex values. Their approach is based on Galerkin approximations, and, consequently, their result depends crucially on the convexity of the values of $F$ and their proof cannot be modified to deal with the nonconvex problem. Similarly for the work of Papageorgiou et al., which uses a surjectivity result for operators of monotone type (see Proposition 2.1 in $\S 2$ ).

To our knowledge, the problem studied here has not been attacked in this generality by any other author. Here we drop the monotonicity hypothesis on $A(t, \cdot)$ and replace $F(t, x)$ by ext $F(t, x)$ (the extreme points of $F(t, x)$ ). Note that ext $F(t, x)$ need not be closed and the multifunction $x \rightarrow \operatorname{ext} F(t, x)$ need not have any continuity properties, even if $F(t, \cdot)$ is 'very good', for example $h$-continuous. We should point out that $\mathrm{Hu}$ and Papageorgiou [9] and $\mathrm{Hu}$ et al. [10] recently examined periodic problems for non-convex differential inclusions in $\mathbb{R}^{N}$. However, they did not address the question of the existence of extremal solutions that we consider here.

\section{Preliminaries and extremal solutions}

In this section we fix our notation and recall some basic definitions and facts from multivalued analysis and the theory of nonlinear operators of monotone type. Details can be found in the books of Hu and Papageorgiou [7] and Zeidler [22].

Let $(\Omega, \Sigma)$ be a measurable space and $X$ a separable Banach space. We will use the following notation:

$$
\begin{aligned}
P_{f(c)}(X) & =\{A \subseteq X: A \text { is non-empty, closed (and convex) }\} \\
P_{(w) k(c)}(X) & =\{A \subseteq X: A \text { is non-empty, (weakly) compact (and convex) }\}
\end{aligned}
$$

A multifunction (set-valued function) $F: \Omega \rightarrow P_{f}(X)$ is said to be 'measurable', if, for all $x \in X$, the function $\omega \rightarrow d(x, F(\omega))=\inf \{\|x-v\|: v \in F(\omega)\}$ is a measurable, $\mathbb{R}_{+}$-valued function. A multifunction $F: \Omega \rightarrow 2^{X} \backslash\{\emptyset\}$ is said to be graph measurable if

$$
\operatorname{Gr} F=\{(\omega, x) \in \Omega \times X: x \in F(\omega)\} \in \Sigma \times \mathcal{B}(X),
$$

with $\mathcal{B}(X)$ being the Borel $\sigma$-field of $X$. For multifunctions with values in $P_{f}(X)$, measurability implies graph measurability, while the converse is true if $\Sigma=\hat{\Sigma}$ with $\hat{\Sigma}$ being the universal $\sigma$ field. Recall that $\Sigma=\hat{\Sigma}$, if there is a $\sigma$-finite measure $\mu$ on $(\Omega, \Sigma)$ with respect to which $\Sigma$ is complete. Given $1 \leqslant p \leqslant \infty$, by $S_{F}^{p}$ we denote the set of all $L^{p}(\Omega, X)$-selectors of $F(\cdot)$; i.e. $S_{F}^{p}=\left\{f \in L^{p}(\Omega, X): f(\omega) \in F(\omega) \mu\right.$-a.e. $\}$. Note that for a graph measurable multifunction $F: \Omega \rightarrow 2^{X} \backslash\{\emptyset\}$, the set $S_{F}^{p}$ is non-empty if and only if the $\mathbb{R}_{+}$-valued function $\omega \rightarrow \inf [\|z\|: z \in F(\omega)]$ belongs to $L^{p}(\Omega)$.

On $P_{f}(X)$ we can define a generalized metric $h(\cdot, \cdot)$, known in the literature as the 'Hausdorff metric', by setting

$$
h(A, B)=\max [\sup \{d(a, B): a \in A\}, \sup \{d(b, A): b \in B\}] .
$$


The metric space $\left(P_{f}(X), h\right)$ is complete and $P_{f c}(X)$ is a closed subspace, while $P_{k}(X)$ is a separable, closed subspace (hence a Polish space). A multifunction $F: X \rightarrow P_{f}(X)$ is said to be Hausdorff continuous ( $h$-continuous) if it is continuous from $X$ into $\left(P_{f}(X), h\right)$.

Let $Y, Z$ be Hausdorff topological spaces. A multifunction $G: Y \rightarrow 2^{Z} \backslash\{\emptyset\}$ is said to be lower semicontinuous (respectively, upper semicontinuous), if, for all $C \subseteq Z$ closed, the set $F^{+}(C)=\{y \in Y: F(y) \subseteq C\}$ (respectively, $F^{-}(C)=\{y \in Y: F(y) \cap C \neq \emptyset\}$ ) is closed.

Next, let $H$ be a Hilbert space and $X$ a dense subspace carrying the structure of a separable reflexive Banach space, which is embedded into $H$ continuously. Identifying $H$ with its dual (pivot space), we have $X \subseteq H \subseteq X^{*}$ with all embeddings being continuous and dense. In this paper we assume that they are also compact. The triple $\left(X, H, X^{*}\right)$ is called 'evolution triple'. By $|\cdot|$ (respectively, $\|\cdot\|,\|\cdot\|_{*}$ ) we denote the norm of $H$ (respectively, of $X$ and $\left.X^{*}\right)$. By $(\cdot, \cdot)$ we denote the inner product of $H$, and by $\langle\cdot, \cdot\rangle$ the duality brackets of the pair $\left(X, X^{*}\right)$. The two are compatible in the sense that $\left.\langle\cdot, \cdot\rangle\right|_{X \times H}=(\cdot, \cdot)$.

Let $1<p, q<\infty,(1 / p)+(1 / q)=1, T=[0, b]$. We define

$$
W_{p q}(T)=\left\{x \in L^{p}(T, X): \dot{x} \in L^{q}\left(T, X^{*}\right)\right\}
$$

The derivative involved in this definition is understood in the sense of vector-valued distributions. Equipped with the norm

$$
\|x\|_{p q}=\left\{\|x\|_{p}^{2}+\|\dot{x}\|_{q}^{2}\right\}^{1 / 2}
$$

the space $W_{p q}(T)$ becomes a separable, reflexive Banach space. It is well known that $W_{p q}(T)$ is embedded continuously in $C(T, H)$ (so every element of $W_{p q}(T)$ has a representative in $C(T, H)$ ). Since in this work we assume that $X$ is embedded compactly in $H$, we also have that $W_{p q}(T)$ is embedded compactly in $L^{p}(T, H)$.

Let $Y$ be a reflexive Banach space, $L: D \subseteq Y \rightarrow Y^{*}$ is a linear, densely defined maximal monotone operator and $K: Y \rightarrow P_{w k c}\left(Y^{*}\right)$. We say that $K(\cdot)$ is 'coercive' if

$$
\frac{\inf \left[(v, y)_{Y^{*}, Y}: v \in K(y)\right]}{\|y\|} \rightarrow \infty, \quad \text { as }\|y\| \rightarrow \infty
$$

We say that $K(\cdot)$ is an ' $L$-generalized pseudomonotone operator' if it is demicontinuous, bounded (i.e. maps bounded sets to bounded ones) and if $\left\{y_{n}\right\}_{n \geqslant 1} \subseteq D$ is such that $y_{n} \stackrel{w}{\rightarrow} y$ in $Y, L\left(y_{n}\right) \stackrel{w}{\rightarrow} L(y)$ in $Y^{*}$, and $\lim \sup \left(K\left(y_{n}\right), y_{n}-y\right)_{Y^{*}, Y} \leqslant 0$, then we have $K\left(y_{n}\right) \stackrel{w}{\rightarrow} K(y)$ in $Y^{*}$ and $\left(K\left(y_{n}\right), y_{n}\right)_{Y^{*}, Y} \rightarrow(K(y), y)_{Y^{*}, Y}$ as $n \rightarrow \infty$. Also we say that the multivalued operator $V$ is ' $L$-generalized pseudomonotone'

(a) if for every $y \in Y, V(y) \in P_{w k c}\left(Y^{*}\right)$;

(b) if $V(\cdot)$ is upper semicontinuous from every finite-dimensional subspace $Z$ of $Y$ into $Y_{w}^{*} ;$ and 
(c) if $\left\{y_{n}\right\}_{n \geqslant 1} \subseteq D$ is such that $y_{n} \stackrel{w}{\rightarrow} y$ in $Y, L\left(y_{n}\right) \stackrel{w}{\rightarrow} L(y)$ in $Y^{*}, y_{n}^{*} \in V\left(y_{n}\right)$, $n \geqslant 1, y_{n}^{*} \stackrel{w}{\rightarrow} y^{*}$ in $Y^{*}$, and $\lim \sup \left(y_{n}^{*}, y_{n}\right)_{Y^{*}, Y} \leqslant\left(y^{*}, y\right)_{Y^{*}, Y}$, then $\left(y, y^{*}\right) \in \operatorname{Gr} V$ and $\left(y_{n}^{*}, y_{n}\right)_{Y^{*}, Y} \rightarrow\left(y^{*}, y\right)_{Y^{*}, Y}$.

The following surjectivity result for $L$-generalized pseudomonotone operators will be used in the main existence theorem.

Proposition 2.1. If $Y$ is a reflexive and strictly convex Banach space, $L: D \subseteq Y \rightarrow$ $Y^{*}$ is a linear densely defined maximal monotone operator and $K: Y \rightarrow 2^{Y^{*}} \backslash\{\emptyset\}$ is a bounded, $L$-generalized pseudomonotone map that is coercive, then $R(L+V)=Y^{*}$.

Remark 2.2. This surjectivity result for $K$ single-valued can be found in Lions [11], while the result for multivalued $K(\cdot)$ can be found in Papageorgiou et al. [17].

Let $T=[0, b]$ and let $\left(X, H, X^{*}\right)$ be an evolution triple of spaces with the embeddings being compact. We examine the following problem:

$$
\left.\begin{array}{cc}
\dot{x}(t)+A(t, x(t)) & \in \operatorname{ext} F(t, x(t)), \quad \text { a.e. on } T, \\
x(0)=x(b) . &
\end{array}\right\}
$$

By ext $F(t, x(t))$ we denote the extreme points of the set $F(t, x(t))$. Our hypotheses on the data of (2.1) follow.

$H(A)_{1}: A: T \times X \rightarrow X^{*}$ is an operator such that

(i) for every $x \in H, t \rightarrow A(t, x)$ is measurable;

(ii) for almost all $t \in T, x \rightarrow A(t, x)$ is demicontinuous, pseudomonotone;

(iii) for almost all $t \in T$ and all $x \in X$ we have

$$
\|A(t, x)\|_{*} \leqslant a_{1}(t)+c_{1}\|x\|^{p-1},
$$

with $a_{1} \in L^{q}(T)(p \geqslant 2,(1 / p)+(1 / q)=1), c_{1}>0 ;$

(iv) for almost all $t \in T$ and all $x \in X$ we have

$$
\langle A(t, x), x\rangle \geqslant c\|x\|^{p}-a(t), \quad \text { with } a \in L^{1}(T)_{+}, \quad c>0 .
$$

$H(F)_{1}: F: T \times H \rightarrow P_{f c}(H)$ is a multifunction such that

(i) for every $x \in H, t \rightarrow F(t, x)$ is measurable;

(ii) for all $t \in T, x \rightarrow F(t, x)$ is $h$-continuous;

(iii) for almost all $t \in T$ and all $x \in H$, we have

$$
|F(t, x)|=\sup \{|y|: y \in F(t, x)\} \leqslant a_{2}(t)+c_{2}|x|^{2 / q}
$$

with $a_{2} \in L^{q}(T), c_{2}>0$;

(iv) there exists $r>0$ such that for almost all $t \in T$, all $|x|=r$ and all $y \in F(t, x)$, we have $(y, x) \leqslant-a(t)$. 
By a solution of (2.1) we mean a function $x \in W_{p q}(T)$ such that $\dot{x}(t)+A(t, x(t))=$

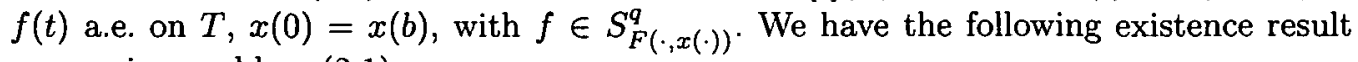
concerning problem (2.1).

Theorem 2.3. If hypotheses $H(A)_{1}$ and $H(F)_{1}$ hold, then problem (2.1) has a solution $x \in W_{p q}(T)$.

Proof. We introduce a modification of the orientor field $F(t, x)$. So let $F_{1}: T \times H \rightarrow$ $P_{f c}(H)$ be defined by

$$
F_{1}(t, x)= \begin{cases}F(t, x), & \text { if }|x| \leqslant r \\ -\left(x-p_{r}(x)\right)+F\left(t, p_{r}(x)\right), & \text { if }|x| \geqslant r\end{cases}
$$

where $p_{r}(\cdot)$ is the $r$-radial retraction on $H$; i.e.

$$
p_{r}(x)= \begin{cases}x, & \text { if }|x| \leqslant r \\ r x /|x|, & \text { if }|x|>r\end{cases}
$$

Recall that $p_{r}(\cdot)$ is Lipschitz continuous. Then it is clear from the above definition that $t \rightarrow F_{1}(t, x)$ is measurable and $x \rightarrow F_{1}(t, x)$ is $h$-continuous. Moreover, $\left|F_{1}(t, x)\right| \leqslant$ $a_{3}(t)+c_{3}|x|^{2 / q}$ a.e. on $T$, for all $x \in H$ and with $a_{3} \in L^{q}(T), c_{3}>0$. Consider the following periodic problem:

$$
\left.\begin{array}{c}
\dot{x}(t)+A(t, x(t)) \in F_{1}(t, x(t)), \quad \text { a.e. on } T, \\
x(0)=x(b) .
\end{array}\right\}
$$

We will show that (2.2) has a solution $x \in W_{p q}(T)$. To this end let $L: D \subseteq L^{p}(T, X) \rightarrow$ $L^{q}\left(T, X^{*}\right)$ be defined by $L(x)=\dot{x}$ for all $x \in D=\left\{x \in W_{p q}(T): x(0)=x(b)\right\}$. It is well known that $L$ is linear, densely defined and maximal monotone (see, for example, Hu and Papageorgiou [7, p. 419] or Zeidler [22, p. 855]). Let $\hat{A}: L^{p}(T, X) \rightarrow L^{q}\left(T, X^{*}\right)$ be the Nemitsky operator corresponding to the map $A(t, x)$, i.e. $\hat{A}(x)(\cdot)=A(\cdot, x(\cdot))$. Also, $G_{1}: L^{p}(T, H) \rightarrow P_{f c}\left(L^{q}(T, H)\right)$ the multivalued Nemitsky operator corresponding to $-F_{1}(t, x)$, i.e. $G_{1}(x)=\left\{g \in L^{q}(T, H): g(t) \in-F_{1}(t, x)\right.$ a.e. on $\left.T\right\}$.

Claim 2.4. $\hat{A}+G_{1}$ is $L$-generalized pseudomonotone.

First we show that $\hat{A}+G_{1}: L^{p}(T, X) \rightarrow P_{w k c}\left(L^{q}\left(T, X^{*}\right)\right)$ is upper semicontinuous from $L^{p}(T, X)$ into $L^{q}\left(T, X^{*}\right)_{w}$. To this end we have to show that for every $C \subseteq L^{q}\left(T, X^{*}\right)$ nonempty and weakly closed, the set $\left(\hat{A}+G_{1}\right)^{-}(C)=\left\{x \in L^{p}(T, X):\left(\hat{A}(x)+G_{1}(x)\right) \cap C \neq \emptyset\right\}$ is closed. So let $x_{n} \in\left(\hat{A}+G_{1}\right)^{-}(C), n \geqslant 1$, and assume that $x_{n} \rightarrow x$ in $L^{p}(T, X)$ as $n \rightarrow \infty$. Let $u_{n} \in\left(\hat{A}+G_{1}\right)\left(x_{n}\right) \cap C$. Evidently, $\left\{u_{n}\right\}_{n \geqslant 1} \subseteq L^{q}\left(T, X^{*}\right)$ is bounded, and so, by passing to a subsequence, we may assume that $u_{n} \stackrel{w}{\rightarrow} u$ in $L^{q}\left(T, X^{*}\right)$ as $n \rightarrow \infty$. We have $u_{n}=\hat{A}\left(x_{n}\right)+g_{n}$, with $g_{n} \in G_{1}\left(x_{n}\right), n \geqslant 1$. The sequence $\left\{g_{n}\right\}_{n \geqslant 1} \subseteq L^{q}(T, H)$ is bounded and so we may assume that $g_{n} \stackrel{w}{\longrightarrow} g$ in $L^{q}(T, H)$. Using Proposition VII.3.9 from $\mathrm{Hu}$ and Papageorgiou [7, p. 694], we have that $g \in G_{1}(x)$. Also, if by $((\cdot, \cdot))$ (respectively, $\left.(\cdot, \cdot)_{p q}\right)$ we denote the duality brackets for the pair $\left(L^{p}(T, X), L^{q}\left(T, X^{*}\right)=L^{p}(T, X)^{*}\right)$ 
(respectively, for the pair $\left.L^{p}(T, H), L^{q}(T, H)=L^{p}(T, H)^{*}\right)$, we have $\left(\left(g_{n}, x_{n}-x\right)\right)=$ $\left(g_{n}, x_{n}-x\right)_{p q} \rightarrow 0$ as $n \rightarrow \infty$. In addition we have $\left(\left(u_{n}, x_{n}-x\right)\right) \rightarrow 0$ as $n \rightarrow \infty$. So we obtain $\overline{\lim }\left(\left(\hat{A}\left(x_{n}\right), x_{n}-x\right)\right) \leqslant 0$. But, from Proposition 1 of Papageorgiou [16], we know that $\hat{A}$ is $L$-generalized pseudomonotone. Hence, $\hat{A}\left(x_{n}\right) \stackrel{w}{\rightarrow} \hat{A}(x)$ in $L^{q}\left(T, X^{*}\right)$, and so $u=\hat{A}(x)+g$ with $g \in G_{1}(x)$. Thus, $u \in\left(\hat{A}+G_{1}(x)\right) \cap C$, which proves the upper semicontinuity of $\hat{A}+G_{1}$.

Next let $\left\{x_{n}\right\}_{n \geqslant 1} \subseteq D$ and assume that $x_{n} \stackrel{w}{\rightarrow} x$ in $L^{p}(T, X), x \in D, L\left(x_{n}\right) \stackrel{w}{\rightarrow} L(x)$ in $L^{q}\left(T, X^{*}\right)$ (hence, $x_{n} \stackrel{w}{\rightarrow} x$ in $W_{p q}(T)$ as $\left.n \rightarrow \infty\right), x_{n}^{*} \in\left(\hat{A}+G_{1}\right)\left(x_{n}\right), n \geqslant 1, x_{n}^{*} \stackrel{w}{\rightarrow} x^{*}$ in $L^{q}\left(T, X^{*}\right)$ and $\varlimsup \lim \left(\left(x_{n}^{*}, x_{n}-x\right)\right) \leqslant 0$. By definition we have $x_{n}^{*}=\hat{A}\left(x_{n}\right)+g_{n}$ with $g_{n} \in G_{1}\left(x_{n}\right), n \geqslant 1$. As before we may assume that $g_{n} \stackrel{w}{\longrightarrow} g$ in $L^{q}(T, H)$ and we can check that $g \in G_{1}(x)$ (recall that $x_{n} \stackrel{w}{\rightarrow} x$ in $W_{p q}(T)$ implies that $x_{n} \rightarrow x$ in $L^{p}(T, H)$ ). So we have

$$
\begin{aligned}
& \varlimsup \\
& \Rightarrow \hat{A}\left(x_{n}\right) \stackrel{w}{\rightarrow} \hat{A}(x), \quad \text { in } L^{q}\left(T, X^{*}\right) \quad \text { and } \quad\left(\left(\hat{A}\left(x_{n}\right), x_{n}\right)\right) \rightarrow((\hat{A}(x), x))
\end{aligned}
$$

(from the $L$-generalized pseudomonotonicity of $\hat{A}(\cdot)$ ),

$$
\Rightarrow x^{*}=\hat{A}(x)+g, \quad \text { with } g \in G_{1}(x) \quad \text { and } \quad\left(\left(x_{n}^{*}, x_{n}\right)\right) \rightarrow\left(\left(x^{*}, x\right)\right), \quad \text { as } n \rightarrow \infty,
$$

$\Rightarrow \hat{A}+G_{1}$ is $L$-generalized pseudomonotone as claimed.

Claim 2.5. $\hat{A}+G_{1}$ is coercive.

Let $x \in L^{p}(T, X)$ and $x^{*} \in\left(\hat{A}+G_{1}\right)(x)$. So $x^{*}=\hat{A}(x)+g$ with $g \in G_{1}(x)$. We have

$$
((\hat{A}(x), x)) \geqslant c\|x\|_{p}^{p}-\|a\|_{1} \text {. }
$$

Also,

$$
((g, x))=(g, x)_{p q}=\int_{0}^{b}(g(t), x(t)) \mathrm{d} t=\int_{|x| \geqslant r}(g(t), x(t)) \mathrm{d} t+\int_{|x|<r}(g(t), x(t)) \mathrm{d} t .
$$

On the set $\{t \in T:|x(t)| \geqslant r\}$ we have that $-g(t)=-\left(x(t)-p_{r}(x(t))\right)+f(t)$ a.e. on $T$ with $f(t) \in F\left(t, p_{r}(x(t))\right)$ a.e. on $T$. So, using hypothesis $H(F)_{1}$ (iv) we obtain

$$
\begin{aligned}
-((g, x)) & \leqslant \int_{|x| \geqslant r}-\left(x(t)-p_{r}(x(t)), x(t)\right) \mathrm{d} t+\int_{|x|<r}(g(t), x(t)) \mathrm{d} t \\
& \leqslant \int_{|x|<r}|g(t)| r \mathrm{~d} t \leqslant r\|g\|_{1} \leqslant r b^{1 / p}\|g\|_{q}=c_{4}\|g\|_{g} \quad\left(\text { with } c_{4}=r b^{1 / p}\right) .
\end{aligned}
$$

Thus, finally, we can write that

$$
\left(\left(x^{*}, x\right)\right)=((\hat{A}(x), x))+((g, x)) \geqslant c\|x\|_{p}^{p}-\|a\|_{1}-c_{4}\|g\|_{q}
$$

$\Rightarrow \hat{A}+G_{1}$ is coercive as claimed. 
Claims 2.4 and 2.5 allow us to apply Proposition 2.1 and obtain $x \in W_{p q}(T)$ such that $0 \in\left(L+A+G_{1}\right)(x) \Rightarrow \dot{x}(t)+A(t, x(t)) \in F_{1}(t, x(t))$ a.e. on $T$ and $x(0)=x(b)$, i.e. $x \in W_{p q}(T)$ is a solution of problem (2.2).

Claim 2.6. For every solution $x \in W_{p q}(T)$ of (2.2) and every $t \in T$, we have $|x(t)| \leqslant r$.

Suppose that $|x(t)|>r$ for all $t \in(\beta, \gamma)$ and $|x(\beta)|=|x(\gamma)|=r$. We have $\langle\dot{x}(t), x(t)\rangle+$ $\langle A(t, x(t)), x(t)\rangle=(g(t), x(t))$ a.e. on $[\beta, \gamma]$ with $g \in S_{F_{1}(\cdot, x(\cdot))}^{q}$ :

$$
\begin{array}{ll}
\Rightarrow \frac{1}{2} \frac{\mathrm{d}}{\mathrm{d} t}|x(t)|^{2} \leqslant-\left(x(t)-p_{r}(x(t)), x(t)\right)+(f(t), x(t))+a(t), & \text { a.e. on }[\beta, \gamma], \\
& \text { with } f \in S_{F\left(\cdot, p_{r}(x(\cdot))\right)}^{q}, \\
\Rightarrow \frac{1}{2} \frac{\mathrm{d}}{\mathrm{d} t}|x(t)|^{2} \leqslant-|x(t)|^{2}+\frac{r}{|x(t)|}|x(t)|^{2}+(f(t), x(t))+a(t)<0, & \text { a.e. on }[\beta, \gamma]
\end{array}
$$

(see hypothesis $H(F)_{1}$ (iv)).

Integrating over $[\beta, \gamma]$ we obtain $|x(\gamma)|<|x(\beta)|$ a contradiction. This proves the claim. This claim allows us to assume, without loss of generality, that $\left|F_{1}(t, x)\right| \leqslant \varphi(t)=$ $a_{1}(t)+c_{1} r^{p-1}$ a.e. on $T$ and $\varphi \in L^{q}(T)$. Let

$$
V=\left\{g \in L^{q}(T, H):|g(t)| \leqslant \varphi(t) \text { a.e. on } T\right\} \text {. }
$$

Furnished with the weak topology, this space is compact metrizable. For fixed $g \in V$ consider the following problem:

$$
\left.\begin{array}{rl}
\dot{x}(t)+A(t, x(t)) & =g(t), \quad \text { a.e. on } T, \\
x(0) & =x(b) .
\end{array}\right\}
$$

From the previous arguments we know that the solution set $U(g) \subseteq W_{p q}(T)$ of (2.3) is non-empty. Moreover, if $x \in U(g)$ we have $x \in(L+\hat{A})^{-1}(g)$. But we already know that

$$
(L+\hat{A}): D \subseteq L^{p}(T, X) \rightarrow L^{q}\left(T, X^{*}\right)
$$

is coercive, hence $(L+\hat{A})^{-1}$ maps bounded sets into bounded sets. Therefore, $(L+$ $\hat{A})^{-1}(V)$ is bounded. Then, for $x \in(L+\hat{A})^{-1}(V)$, we have

$$
\begin{aligned}
&\|\dot{x}(t)\|_{*} \leqslant|g(t)|+\|A(t, x(t))\|_{*} \\
& \leqslant \varphi(t)+a_{1}(t)+c_{1}\|x(t)\|^{p-1}, \quad \text { a.e. on } T, \\
& \Rightarrow\|\dot{x}\|_{q} \leqslant\|\varphi\|_{q}+\left\|a_{1}\right\|_{q}+\|x\|_{p}^{p-1}, \\
& \Rightarrow(L+\hat{A})^{-1}(V)=U(V) \subseteq D \subseteq W_{p q}(T), \quad \text { is bounded. }
\end{aligned}
$$

Claim 2.7. $U(V)$ is compact in $C(T, H)$. 
Let $\left\{x_{n}\right\}_{n \geqslant 1} \subseteq U(V)$. We have $x_{n} \in U\left(g_{n}\right), n \geqslant 1$. We may assume that $g_{n} \stackrel{w}{\rightarrow} g$ in $L^{q}(T, H)$ and $x_{n} \stackrel{w}{\rightarrow} x$ in $W_{p q}(T)$, hence $x_{n} \rightarrow x$ in $L^{p}(T, H)$ (recall that $W_{p q}(T)$ is embedded compactly in $\left.L^{p}(T, H)\right)$. Thus we may also assume that $x_{n}(t) \rightarrow x(t)$ in $H$ for all $t \in T \backslash N$, with $|N|=0$ (with $|\cdot|$ denoting the Lebesgue measure on $T$ ). The sequence $\left\{\left\langle\dot{x}_{n}(\cdot), x_{n}(\cdot)-x(\cdot)\right\rangle\right\}_{n \geqslant 1}$ is uniformly integrable. So, given $\varepsilon>0$, we can find $\tau, t \in T \backslash N$ such that

$$
\int_{0}^{\tau}\left|\left\langle\dot{x}_{n}(s), x_{n}(s)-x(s)\right\rangle\right| \mathrm{d} s \leqslant \frac{1}{2} \varepsilon, \quad \int_{t}^{b}\left|\left\langle\dot{x}_{n}(s), x_{n}(s)-x(s)\right\rangle\right| \mathrm{d} s \leqslant \frac{1}{2} \varepsilon .
$$

Extending the notational convention introduced earlier, by $((\cdot, \cdot))_{\tau t}$ we denote the duality brackets for the pair $\left(L^{p}([\tau, t], X), L^{q}\left([\tau, t], X^{*}\right)=L^{p}([\tau, t], X)^{*}\right)$. We have

$$
\left(\left(\dot{x}_{n}, x_{n}-x\right)\right)_{\tau t}=\frac{1}{2}\left|x_{n}(t)-x(t)\right|^{2}-\frac{1}{2}\left|x_{n}(\tau)-x(\tau)\right|^{2}+\left(\left(\dot{x}, x_{n}-x\right)\right)_{\tau t}
$$

and $\frac{1}{2}\left|x_{n}(\tau)-x(\tau)\right|^{2}, \frac{1}{2}\left|x_{n}(t)-x(t)\right|^{2} \rightarrow 0$ (since $\left.\tau, t \in T \backslash N\right),\left(\left(\dot{x}, x_{n}-x\right)\right)_{\tau t} \rightarrow 0$ as $n \rightarrow \infty$. So $\left(\left(\dot{x}_{n}, x_{n}-x\right)\right)_{\tau t} \rightarrow 0$ as $n \rightarrow \infty$. Note that

$$
\begin{aligned}
\left(\left(\dot{x}_{n}, x_{n}-x\right)\right) & =\left(\left(\dot{x}_{n}, x_{n}-x\right)\right)_{\tau t}+\int_{[0, \tau] \cup[t, b]}\left\langle\dot{x}_{n}(s), x_{n}(s)-x(s)\right\rangle \mathrm{d} s \\
\Rightarrow\left(\left(\dot{x}_{n}, x_{n}-x\right)\right) & \geqslant\left(\left(\dot{x}_{n}, x_{n}-x\right)\right)_{\tau t}-\varepsilon \quad(\text { see }(2.4)) \\
\Rightarrow & \underline{\lim }\left(\left(\dot{x}_{n}, x_{n}-x\right)\right) \geqslant-\varepsilon .
\end{aligned}
$$

Let $\varepsilon \downarrow 0$ to conclude that

$$
\underline{\lim }\left(\left(\dot{x}_{n}, x_{n}-x\right)\right) \geqslant 0 .
$$

Employing a similar argument we can show that $\left(\left(\dot{x}_{n}, x_{n}-x\right)\right) \leqslant\left(\left(\dot{x}_{n}, x_{n}-x\right)\right)_{\tau t}+\varepsilon$. Passing to the limit as $n \rightarrow \infty$ and then letting $\varepsilon \downarrow 0$, we obtain

$$
\varlimsup \overline{\lim }\left(\left(\dot{x}_{n}, x_{n}-x\right)\right) \leqslant 0 .
$$

From (2.5) and (2.6) we have that $\left(\left(\dot{x}_{n}, x_{n}-x\right)\right) \rightarrow 0$. Since

$$
\left(\left(\dot{x}_{n}, x_{n}-x\right)\right)=\left(\left(\hat{A}\left(x_{n}\right), x_{n}-x\right)\right)+\left(g_{n}, x_{n}-x\right)_{p q},
$$

we infer that $\varlimsup \lim \left(\left(\hat{A}\left(x_{n}\right), x_{n}-x\right)\right) \leqslant 0$. Because $\hat{A}$ is $L$-generalized pseudomonotone (see Papageorgiou [16]), we have that $\hat{A}\left(x_{n}\right) \stackrel{w}{\longrightarrow} \hat{A}(x)$ in $L^{q}\left(T, X^{*}\right)$ and $\left(\left(\hat{A}\left(x_{n}\right), x_{n}\right)\right) \rightarrow$ $((\hat{A}(x), x))$ as $n \rightarrow \infty$. In the limit as $n \rightarrow \infty$, we have $\dot{x}(t)+A(t, x(t))=g(t)$ a.e. on $T$, $x(0)=x(b)$ and $g \in V$. So $x \in U(K)$. Also, for every $t \in T$

$$
\begin{aligned}
\frac{1}{2}\left|x_{n}(t)-x(t)\right|^{2} & =\left(\left(g_{n}-g, x_{n}-x\right)\right)_{t}-\left(\left(\hat{A}\left(x_{n}\right)-\hat{A}(x), x_{n}-x\right)\right)_{t} \\
\frac{1}{2}\left|x_{n}(t)-x(t)\right|^{2} \leqslant & \int_{0}^{b}\left|\left(g_{n}(s)-g(s), x_{n}(s)-x(s)\right)\right| \mathrm{d} s \\
& +\int_{0}^{b}\left|\left\langle A\left(s, x_{n}(s)\right), x_{n}(s)-x(s)\right\rangle\right| \mathrm{d} s+\left(\left(\hat{A}(x), x_{n}-x\right)\right)_{t} .
\end{aligned}
$$


Note that

$$
\int_{0}^{b}\left|\left(g_{n}(s)-g(s), x_{n}(s)-x(s)\right)\right| \mathrm{d} s \rightarrow 0, \quad \text { as } n \rightarrow \infty
$$

Also, if

$$
h_{n}(t)=\left\langle A\left(t, x_{n}(t)\right), x_{n}(t)-x(t)\right\rangle
$$

from the proof of Proposition 1 of Papageorgiou [16], we know that $h_{n} \rightarrow 0$ in $L^{1}(T)$. So

$$
\int_{0}^{b}\left|\left\langle A\left(t, x_{n}(t)\right), x_{n}(t)-x(t)\right\rangle\right| \mathrm{d} t \rightarrow 0, \quad \text { as } n \rightarrow \infty
$$

Moreover, let

$$
\eta_{n}(t)=\int_{0}^{t}\left\langle A(s, x(s)), x_{n}(s)-x(s)\right\rangle \mathrm{d} s
$$

Let $t_{n} \in T$ such that

$$
\eta_{n}\left(t_{n}\right)=\max \left[\eta_{n}(t): t \in T\right]
$$

We may assume that $t_{n} \rightarrow t \in T$. We have

$$
\eta_{n}\left(t_{n}\right)=\int_{0}^{t_{n}}\left\langle A(s, x(s)), x_{n}(s)-x(s)\right\rangle \mathrm{d} s=\left(\left(\chi_{\left[0, t_{n}\right]} \hat{A}(x), x_{n}-x\right)\right) .
$$

Note that

$$
\int_{0}^{b}\left\|\chi_{\left[0, t_{n}\right]}(s) A(s, x(s))-\chi_{[0, t]}(s) A(s, x(s))\right\| \mathrm{d} s=\int_{t_{n} \wedge t}^{t_{n} \vee t}\|A(s, x(s))\|_{*} \mathrm{~d} s
$$

(here, $t_{n} \vee t=\max \left\{t_{n}, t\right\}$ and $t_{n} \wedge t=\min \left\{t_{n}, t\right\}$ ). But

$$
\begin{array}{rl}
\int_{t_{n} \wedge t}^{t_{n} \vee t}\|A(s, x(s))\|_{*} & \mathrm{~d} s \rightarrow 0, \quad \text { as } n \rightarrow \infty, \\
\Rightarrow \chi_{\left[0, t_{n}\right]} \hat{A}\left(x_{n}\right) \rightarrow \chi_{[0, t]} \hat{A}(x), \quad \text { in } L^{q}\left(T, X^{*}\right), \quad \text { as } n \rightarrow \infty,
\end{array}
$$

$\Rightarrow \eta_{n}\left(t_{n}\right) \rightarrow 0$ as $n \rightarrow \infty$ and so $\sup _{t \in T}\left(\left(\hat{A}(x), x_{n}-x\right)\right)_{t} \rightarrow 0$. Thus, using all these convergences in (2.7), we obtain

$$
\sup _{t \in T}\left|x_{n}(t)-x(t)\right|=\left\|x_{n}-x\right\|_{\infty} \rightarrow 0, \quad \text { as } n \rightarrow \infty
$$

which proves the claim.

Let $K=\overline{\operatorname{conv}} U(V) \in P_{k c}(C(T, H))$ and consider the multifunction

$$
R: K \rightarrow P_{w k c}\left(L^{q}(T, H)\right)
$$

defined by

$$
R(x)=S_{F_{1}(\cdot, x(\cdot))}^{q}
$$


Let $L_{w}^{1}(T, H)$ denote the space $L^{1}(T, H)$ equipped with the 'weak norm'

$$
\|f\|_{w}=\sup \left[\left|\int_{s}^{t} f(\tau) \mathrm{d} \tau\right|: 0 \leqslant s \leqslant t \leqslant b\right] .
$$

Using Theorem II.8.31 from $\mathrm{Hu}$ and Papageorgiou $[7$, p. 260] we can find $r: K \rightarrow$ $L_{w}^{1}(T, H)$, a continuous map such that $r(x) \in \operatorname{ext} R(x)$ for all $x \in K$. We know that

$$
\operatorname{ext} S_{F_{1}(\cdot, x(\cdot))}^{q}=S_{\operatorname{ext} F_{1}(\cdot, x(\cdot))}^{q}
$$

(see Theorem II.4.6 in $\mathrm{Hu}$ and Papageorgiou [7, p. 192]). Note that $K$ viewed as a subset of the uniformly convex space $L^{p}(T, H)$ is compact and convex (recall that $W_{p q}(T)$ is embedded compactly in $\left.L^{p}(T, H)\right)$. So the metric projection map $x \rightarrow p(x ; K)$ from $L^{p}(T, H)$ into $K \subseteq L^{p}(T, H)$ is well defined and continuous. Let

$$
\hat{r}: L^{p}(T, H) \rightarrow L^{q}(T, H)
$$

be defined by $\hat{r}(x)=r(p(x ; K))$.

Claim 2.8. $\hat{A}-\hat{r}$ is $L$-generalized pseudomonotone.

To this end let $x_{n} \in D, n \geqslant 1$, and assume that $x_{n} \stackrel{w}{\rightarrow} x$ in $W_{p q}(T)$ with

$$
\varlimsup \overline{\lim }\left(\left(\hat{A}\left(x_{n}\right)-\hat{r}\left(x_{n}\right), x_{n}-x\right)\right) \leqslant 0 .
$$

We know that $x_{n} \rightarrow x$ in $L^{p}(T, H)$ and so $p\left(x_{n} ; K\right) \rightarrow p(x ; K)$ in $L^{p}(T, H)$ as $n \rightarrow \infty$. Hence,

$$
\hat{r}\left(x_{n}\right) \stackrel{\|\cdot\|_{w}}{\longrightarrow} \hat{r}(x)
$$

which, by virtue of the lemma of Papageorgiou [15], implies that $\hat{r}\left(x_{n}\right) \stackrel{w}{\rightarrow} \hat{r}(x)$ in $L^{q}(T, H)$ as $n \rightarrow \infty$. Also, $\left(\left(\hat{r}\left(x_{n}\right), x_{n}-x\right)\right)=\left(\hat{r}\left(x_{n}\right), x_{n}-x\right)_{p q} \rightarrow 0$ and so $\overline{\lim }\left(\left(\hat{A}\left(x_{n}\right)\right.\right.$, $\left.\left.x_{n}-x\right)\right) \leqslant 0$. As before, we infer that $\hat{A}\left(x_{n}\right) \stackrel{w}{\rightarrow} \hat{A}(x)$ in $L^{q}\left(T, X^{*}\right)$ and $\left(\left(\hat{A}\left(x_{n}\right), x_{n}\right)\right) \rightarrow$ $((\hat{A}(x), x))$. Thus, finally, $\hat{A}\left(x_{n}\right)-\hat{r}\left(x_{n}\right) \stackrel{w}{\rightarrow} \hat{A}(x)-\hat{r}(x)$ in $L^{q}\left(T, X^{*}\right)$ and $\left(\left(\hat{A}\left(x_{n}\right)-\right.\right.$ $\left.\left.\hat{r}\left(x_{n}\right), x_{n}\right)\right) \rightarrow((\hat{A}(x)-\hat{r}(x), x))$ as $n \rightarrow \infty$, which prove the claim.

Claim 2.9. $\hat{A}-\hat{r}$ is coercive.

For every $x \in L^{p}(T, X)$, we have

$$
\begin{aligned}
((\hat{A}(x)-\hat{r}(x), x)) & =((\hat{A}(x), x))-((\hat{r}(x), x)) \\
& \left.\geqslant c\|x\|_{p}^{p}-\|a\|_{1}-c_{5}\|\varphi\|_{q}\|x\|_{p} \quad \text { (for some } c_{5}>0\right)
\end{aligned}
$$

$\Rightarrow \hat{A}-\hat{r}$ is coercive.

Claims 2.8 and 2.9 allow us to use Proposition 2.1 to obtain a solution $x \in W_{p q}(T)$ such that

$$
\begin{gathered}
\dot{x}(t)+A(t, x(t))=r(p(x ; K))(t), \quad \text { a.e. on } T \\
x(0)=x(b) .
\end{gathered}
$$


But $r(p(x ; K)) \in V$, hence $x \in U(V) \subseteq K$ and so $p(x ; K)=x$. Because $r(x) \in$ $S_{\text {ext } F_{1}(\cdot, x(\cdot))}^{q}$ we have $r(x)(t) \in \operatorname{ext} F_{1}(t, x(t))$ a.e. on $T$. Also, from the first part of the proof we have that $|x(t)| \leqslant r$ for all $t \in T$ and so $F_{1}(t, x(t))=F(t, x(t))$. Therefore $x \in W_{p q}(T)$ solves $(2.1)$.

\section{Strong relaxation}

In conjunction with (2.1) we consider the convexified problem

$$
\left.\begin{array}{c}
\dot{x}(t)+A(t, x(t)) \in F(t, x(t)), \quad \text { a.e. on } T, \\
x(0)=x(b) .
\end{array}\right\}
$$

Let $S_{e} \subseteq W_{p q}(T)$ be the solution set of (2.1) and $S_{c} \subseteq W_{p q}(T)$ the solution set of 3.1. We want to have conditions that imply that $S_{c}=\bar{S}_{e} C(T, H)$ ('strong relaxation theorem'). Such a result is of importance in the context of control systems, because it says that any trajectory of the system can be approximated with any degree of accuracy, by trajectories which are generated by bang-bang controls, which are easy to realize.

Now the hypotheses on the data are stronger.

$H(A)_{2}: A: T \times X \rightarrow X^{*}$ is an operator such that

(i) for all $x \in X, t \rightarrow A(t, x)$ is measurable;

(ii) for almost all $t \in T, x \rightarrow A(t, x)$ is demicontinuous, monotone;

(iii) for almost all $t \in T$ and all $x \in X$ we have

$$
\|A(t, x)\|_{*} \leqslant a_{1}(t)+c_{1}\|x\|^{p-1}
$$

with $a_{1} \in L^{q}(T), c_{1}>0 ;$

(iv) for almost all $t \in T$ and all $x \in X$, we have

$$
\langle A(t, x), x\rangle \geqslant c\|x\|^{p}-a(t), \quad \text { with } a \in L^{1}(T)_{+}, \quad c>0 .
$$

$H(F)_{2}: F: T \times H \rightarrow P_{f c}(H)$ is a multifunction such that

(i) for all $x \in H, t \rightarrow F(t, x)$ is measurable;

(ii) for almost all $t \in T$ and all $x, y \in H, h(F(t, x), F(t, y)) \leqslant k(t)|x-y|$ with $k \in L^{1}(T)$

(iii) for almost all $t \in T$ and all $x \in H$, we have

$$
|F(t, x)|=\sup \{|y|: y \in F(t, x)\} \leqslant a_{2}(t)+c_{2}|x|^{2 / q}
$$

with $a_{2} \in L^{q}(T), c_{2}>0$;

(iv) there exists $r>0$ such that for almost all $t \in T$, all $x \in H$ with $|x|=r$ and all $y \in F(t, x)$, we have $(y, x) \leqslant-a(t)$. 
Theorem 3.1. If hypotheses $H(A)_{2}$ and $H(F)_{2}$ hold, then $S_{c}=\overline{S_{e}}$ the closure taken in $C(T, H)$.

Proof. Let $x \in S_{c}$ (see Theorem 2.3). By definition we have

$$
\begin{aligned}
\dot{x}(t)+A(t, x(t)) & =f(t), \quad \text { a.e. on } T \\
x(0) & =x(b)
\end{aligned}
$$

with $f \in S_{F(\cdot x(\cdot))}^{q}$. Let $K \in P_{k c}(C(T, H))$ be as in the proof of Theorem 2.3. Given $y \in K$ and $\varepsilon>0$, let

$$
\Gamma_{\varepsilon}: T \rightarrow 2^{H} \backslash\{\emptyset\}
$$

be defined by

$$
\Gamma_{\varepsilon}(t)=\left\{u \in H:|f(t)-u|<(\varepsilon / 2 M b)+d_{H}(f(t), F(t, y(t))), u \in F(t, y(t))\right\}
$$

with $|K|=\sup \left[\|u\|_{L^{q}(T, H)}: u \in K\right] \leqslant M$. It is easy to see that $\operatorname{Gr} \Gamma_{\varepsilon} \in \mathcal{L} \times \mathcal{B}(H)$, with $\mathcal{L}$ being the Lebesgue $\sigma$-field of $T$. We can apply Theorem II.2.14 from Hu and Papageorgiou [7, p. 158] (the Yankov-von Neumann-Aumann selection theorem) to obtain $u: T \rightarrow H$ a Lebesgue-measurable map such that $u(t) \in \Gamma_{\varepsilon}(t)$ for all $t \in T$. So, if we define

$$
L_{\varepsilon}: K \rightarrow 2^{L^{1}(T, H)}
$$

by

$$
L_{\varepsilon}(y)=\left\{u \in S_{F(\cdot, y(\cdot))}^{1}:|f(t)-u(t)|<(\varepsilon / 2 M b)+d_{H}(f(t), F(t, y(t))) \text { a.e. on } T\right\}
$$

we see that $L_{\varepsilon}$ has non-empty, decomposable values. Moreover, Lemma II.8.3 in $\mathrm{Hu}$ and Papageorgiou [7, p. 239] implies that $L_{\varepsilon}$ is lower semicontinuous, and, hence, so is $y \rightarrow \overline{L_{\varepsilon}(y)}$. Invoking Theorem II.8.7 from $\mathrm{Hu}$ and Papageorgiou [7, p. 245], we obtain $u_{\varepsilon}: K \rightarrow L^{1}(T, H)$ a continuous map such that $u_{\varepsilon}(y) \in \overline{L_{\varepsilon}(y)}$ for all $y \in K$. We have

$$
\left|f(t)-u_{\varepsilon}(y)(t)\right| \leqslant(\varepsilon / 2 M b)+k(t)|x(t)-y(t)|, \quad \text { a.e. on } T .
$$

Also using Theorem II.8.31 from $\mathrm{Hu}$ and Papageorgiou [7, p. 260], we can generate $v_{\varepsilon}: K \rightarrow L_{w}^{\mathbf{1}}(T, H)$, a continuous map such that

$$
v_{\varepsilon}(y) \in \operatorname{ext} S_{F(\cdot, y(\cdot))}^{1}=S_{\operatorname{ext} F(\cdot, y(\cdot))}^{1}
$$

and $\left\|u_{\varepsilon}(y)-v_{\varepsilon}(y)\right\|_{w}<\varepsilon$ for all $y \in K$.

Now let $\varepsilon_{n} \downarrow 0$ and set $u_{n}=u_{\varepsilon_{n}}, v_{n}=v_{\varepsilon_{n}}$. Let $z=x(0)=x(b)$, and for each $n \geqslant 1$ consider the following boundary-value problem:

$$
\left.\begin{array}{rl}
\dot{x}_{n}(t)+A\left(t, x_{n}(t)\right) & =v_{n}\left(x_{n}\right)(t), \quad \text { a.e. on } T, \\
x_{n}(0) & =x_{n}(b)=z .
\end{array}\right\}
$$


Claim 3.2. Problem 3.2 has a unique solution $x \in W_{p q}(T)$.

First assume that $z \in X$ and let $A_{1}(t, x)=A(t, x+z), v_{n}^{1}(x)=v_{n}(x+z)$. It is easy to check that the properties of $A$ and $v_{n}$ are passed to $A_{1}$ and $v_{n}^{1}$, respectively. Consider the problem

$$
\left.\begin{array}{cc}
\dot{y}_{n}(t)+A_{1}\left(t, y_{n}(t)\right)=v_{n}^{1}\left(y_{n}\right)(t), & \text { a.e. on } T, \\
y_{n}(0)=y_{n}(b)=0 . &
\end{array}\right\}
$$

Let

$$
L: D \subseteq L^{p}(T, X) \rightarrow L^{q}\left(T, X^{*}\right)
$$

be defined by $L(x)=\dot{x}$ for all $x \in D=\left\{x \in W_{p q}(T): x(0)=x(b)=0\right\}$. This is a linear, densely defined maximal monotone operator. As in the proof of Theorem 2.3, we can check that $\hat{A}_{1}-v_{n}^{1}: L^{p}(T, X) \rightarrow L^{q}\left(T, X^{*}\right)$ is $L$-generalized pseudomonotone and coercive. So we can use Proposition 2.1 and obtain a solution $y_{n} \in W_{p q}(T)$ for problem (3.3). Then $x_{n}(\cdot)=y_{n}(\cdot)+z \in W_{p q}(T)$ is a solution of (3.2) when $z \in X$. Next, let $z \in H$ and let $z_{m} \in X$ such that $z_{m} \rightarrow z$ in $H$ as $m \rightarrow \infty$. Let $x_{n m} \in W_{p q}(T)$ be the solution of (3.2) when the boundary value is $z_{m}$. Then, by standard a priori estimation (see, for example, Papageorgiou [14]), since $\left|z_{m}\right| \leqslant M_{1}$ for all $m \geqslant 1$, we can check that $\left\{x_{n m}\right\}_{m \geqslant 1} \subseteq W_{p q}(T)$ is bounded. Thus, we may assume that $x_{n m} \stackrel{w}{\rightarrow} x_{n}$ in $W_{p q}(T)$ as $m \rightarrow \infty$. As we did in the proof of Theorem 2.3, exploiting the $\hat{L}$-generalized pseudomonotonicity of $\hat{A}$ and the continuity of

$$
v_{n}: L^{p}(T, H) \rightarrow L^{q}(T, H)_{w}
$$

for all $n \geqslant 1$, we obtain $\dot{x}_{n}(t)+A\left(t, x_{n}(t)\right)=v_{n}\left(x_{n}\right)(t)$ a.e. on $T, x_{n}(0)=x_{n}(b)=z$. So problem (3.2) has a solution $x_{n} \in W_{p q}(T)$, which is easily seen to be unique because $A(t, \cdot)$ is monotone.

Since $\left\{x_{n}\right\}_{\geqslant 1} \subseteq K$ and the latter is compact in $C(T, H)$, we may assume that $x_{n} \rightarrow y$ in $C(T, H)$. Since $\left|x_{n}(0)-x(0)\right|=0$, exploiting the monotonicity of $A(t, \cdot)$, we obtain

$$
\begin{aligned}
\frac{1}{2}\left|x_{n}(t)-x(t)\right|^{2} & \leqslant \int_{0}^{t}\left(v_{n}\left(x_{n}\right)(s)-f(s), x_{n}(s)-x(s)\right) \mathrm{d} s \\
& \leqslant \int_{0}^{t}\left(v_{n}\left(x_{n}\right)(s)-u_{n}\left(x_{n}\right)(s), x_{n}(s)-x(s)\right) \mathrm{d} s \\
& \quad+\int_{0}^{t}\left|u_{n}\left(x_{n}\right)(s)-f(s)\right|\left|x_{n}(s)-x(s)\right| \mathrm{d} s .
\end{aligned}
$$

Note that $\left\|v_{n}\left(x_{n}\right)-u_{n}\left(x_{n}\right)\right\|_{w}<\varepsilon_{n} \rightarrow 0$, hence $v_{n}\left(x_{n}\right)-u_{n}\left(x_{n}\right) \stackrel{w}{\rightarrow} 0$ in $L^{q}(T, H)$. Also, by construction, we have

$$
\int_{0}^{t}\left|u_{n}\left(x_{n}\right)(s)-f(s)\right|\left|x_{n}(s)-x(s)\right| \mathrm{d} s \leqslant \varepsilon_{n}+\int_{0}^{t} k(s)\left|x_{n}(s)-x(s)\right|^{2} \mathrm{~d} s .
$$


Passing to the limit as $n \rightarrow \infty$ in (3.4), we obtain

$$
\frac{1}{2}|y(t)-x(t)|^{2} \leqslant \int_{0}^{t} k(s)|y(s)-x(s)|^{2} \mathrm{~d} s
$$

$\Rightarrow x=y$ (via Gronwall's inequality).

So $x_{n} \rightarrow x$ in $C(T, H)$ as $n \rightarrow \infty$ and note that $x_{n} \in S_{e}, n \geqslant 1$. Therefore, $S_{c} \subseteq \overline{S_{e}} C(T, H)$. It is easy to check that $S_{c}$ is closed in $C(T, H)$ and so $S_{c}=\overline{S_{e}}$, the closure in $C(T, H)$.

\section{Examples}

In this section we present two examples illustrating the applicability of our work. So, let $T=[0, b]$ and $Z \subseteq \mathbb{R}^{N}$ a bounded domain with $C^{1}$-boundary $\Gamma$. First we consider a vector-valued nonlinear problem with a multivalued right-hand side. In what follows for a function $x: Z \rightarrow \mathbb{R}^{m}$ we write

$$
\mathrm{D} x=\left(\frac{\partial x_{i}}{\partial z_{k}}\right)_{i=1, k=1}^{m, N}
$$

Also, if $A \in \mathbb{R}^{m N}$, then we define the norm $\|A\|=\operatorname{tr}\left(A A^{t}\right)^{1 / 2}$. Let $x=\left(x_{i}\right)_{i=1}^{m}$ and let $\Delta_{p}^{m}$ be the vectorial $p$-Laplacian, i.e. $\Delta_{p}^{m}(x)=\left(\operatorname{div}\|\mathrm{D} x\|^{p-2} \mathrm{D} x_{i}\right)_{i=1}^{m}$. We consider the following problem:

$$
\left.\begin{array}{c}
\frac{\partial x}{\partial t}-\Delta_{p}^{m} x+a_{0}(t, z, x) \in \operatorname{ext} f(t, z, x), \quad \text { on } T \times Z, \\
\left.x\right|_{T \times \Gamma}=0, \quad x(0, z)=x(b, z), \quad \text { a.e. on } Z .
\end{array}\right\}
$$

Such problems encompass a variety of systems that arise in applications, like closedloop control systems, implicit equations, systems with uncertainties, etc. By a weak solution of (4.1) we mean a function $x \in L^{p}\left(T, W_{0}^{1, p}\left(Z, \mathbb{R}^{m}\right)\right)$ such that

$$
\frac{\partial x}{\partial t} \in L^{q}\left(T, W^{-1, q}\left(Z, \mathbb{R}^{m}\right)\right)
$$

and, for all $\varphi \in L^{p}\left(T, W_{0}^{1, p}\left(Z, \mathbb{R}^{m}\right)\right)$, we have

$$
\begin{aligned}
\left(\left(\frac{\partial x}{\partial t}, \varphi\right)\right)+a(x, \varphi)+\int_{0}^{b} \int_{Z}\left(a_{0}(t, z, x, \mathrm{D} x), \varphi(t, z)\right)_{\mathbb{R}^{m}} \mathrm{~d} z \mathrm{~d} t & \\
& =\int_{0}^{b} \int_{Z}(h(t, z), \varphi(t, z))_{\mathbb{R}^{m}} \mathrm{~d} z \mathrm{~d} t
\end{aligned}
$$

where

$$
a(x, \varphi)=\int_{0}^{b} \int_{Z} \sum_{i=1}^{m}\|\mathrm{D} x\|^{p-2}\left(\mathrm{D} x_{i}, D \varphi_{i}\right)_{\mathbb{R}^{N}} \mathrm{~d} z
$$

and $h \in L^{p}\left(T \times Z, \mathbb{R}^{m}\right)$ with $h(t, z) \in \operatorname{ext} f(t, z, x, \mathrm{D} x)$. Our hypotheses on the data of (4.1) are as follows. 
$H\left(a_{0}\right): a_{0}: T \times Z \times \mathbb{R}^{m} \rightarrow \mathbb{R}^{m}$ is a function such that

(i) for all $x \in \mathbb{R}^{m},(t, z) \rightarrow a_{0}(t, z, x)$ is measurable;

(ii) for all $(t, z) \in T \times Z, x \rightarrow a_{0}(t, z, x)$ is continuous;

(iii) for almost all $(t, z) \in T \times Z$ and all $x \in \mathbb{R}^{m}$, we have $\left\|a_{0}(t, z, x)\right\| \leqslant \beta_{1}(t, z)+$ $c_{1}\|x\|^{p-1}$ with $\beta_{1} \in L^{q}(T \times Z), c_{1}>0$ and $\left(a_{0}(t, z, x), x\right)_{\mathbb{R}^{m}} \geqslant 0$.

$H(f)_{1}: f: T \times Z \times \mathbb{R}^{m} \rightarrow P_{k c}\left(\mathbb{R}^{m}\right)$ is a multifunction such that

(i) for all $x \in \mathbb{R}^{m},(t, z) \rightarrow f(t, z, x)$ is measurable;

(ii) for all $(t, z) \in T \times Z, x \rightarrow f(t, z, x)$ is $h$-continuous;

(iii) for almost all $(t, z) \in T \times Z$ and all $x \in \mathbb{R}^{m},|f(t, z, x)| \leqslant \beta_{2}(t, z)+c_{2}\|x\|^{p-1}$ with $\beta_{2} \in L^{q}(T \times Z), c_{2}>0$

(iv) for almost all $(t, z) \in T \times Z$, all $x \in \mathbb{R}^{m}$ and all $y \in f(t, z, x),(y, x)_{\mathbb{R}^{m}} \leqslant 0$.

Let $X=W_{0}^{1, p}\left(Z, \mathbb{R}^{m}\right), H=L^{2}\left(Z, \mathbb{R}^{m}\right)$ and $X^{*}=W^{-1, q}\left(Z, \mathbb{R}^{m}\right)$. Then $\left(X, H, X^{*}\right)$ is an evolution triple with compact embeddings (Sobolev Embedding Theorem). Let $A_{1}: X \rightarrow X^{*}$ be defined by

$$
\left\langle A_{1}(x), y\right\rangle=\sum_{i=1}^{m} \int_{Z}\|\mathrm{D} x\|^{p-2}\left(\mathrm{D} x_{i}, \mathrm{D} y_{i}\right)_{\mathbb{R}^{N}} \mathrm{~d} z, \quad \text { for all } x, y \in W_{0}^{1, p}\left(Z, \mathbb{R}^{m}\right) .
$$

It is easy to see that $A_{1}(\cdot)$ is monotone, demicontinuous, hence maximal monotone. Also, let $\hat{A}_{2}: T \times X \rightarrow X^{*}$ be defined by $\hat{A}_{2}(t, x)(\cdot)=a_{0}(t, \cdot, x(\cdot)) \in L^{q}\left(Z, \mathbb{R}^{m}\right) \subseteq X^{*}$. Evidently, $t \rightarrow \hat{A}_{2}(t, x)$ is measurable, while from the compact embeddings of $X$ in $L^{p}\left(Z, \mathbb{R}^{m}\right)$ and of $L^{q}\left(Z, \mathbb{R}^{m}\right)$ in $X^{*}$ and from Krasnoselskii's theorem about the continuity of the Nemitsky operator, we infer that $\hat{A}_{2}(t, \cdot)$ is completely continuous. So, if $A(t, x)=$ $A_{1}(x)+\hat{A}_{2}(t, x)$, then $t \rightarrow A(t, x)$ is measurable, while $x \rightarrow A(t, x)$ is pseudomonotone, demicontinuous.

Next let $F: T \times H \rightarrow P_{w k c}(H)$ be defined by

$$
F(t, x)=S_{f(t, \cdot, x(\cdot))}^{2} .
$$

Using hypotheses $H\left(a_{0}\right)$ (iv) and $H(f)_{1}$, we check that $F$ satisfies conditions $H(F)_{1}$. Then we can rewrite (4.1) in the following equivalent evolution inclusion form:

$$
\begin{gathered}
\dot{x}(t)+A(t, x(t)) \in \operatorname{ext} F(t, x(t)), \\
x(0)=x(b) .
\end{gathered}
$$

We can apply Theorem 2.3 and obtain a solution for (4.2), and, hence, for (4.1) too. Thus we can state the following existence theorem for problem (4.1).

Theorem 4.1. If hypotheses $H\left(a_{0}\right)$ and $H(f)_{1}$ hold, then problem (4.1) has a solution $x \in L^{p}\left(T, W_{0}^{1, p}\left(Z, \mathbb{R}^{m}\right)\right) \cap C\left(T, L^{2}\left(Z, \mathbb{R}^{m}\right)\right)$ with $\partial x / \partial t \in L^{q}\left(T, W^{-1, q}\left(Z, \mathbb{R}^{m}\right)\right)$. 
Remark 4.2. If we assume an $h$-Lipschitz condition on $f(t, z, \cdot)$, we can have a strong relaxation theorem.

In the second example we consider a controlled nonlinear Navier-Stokes equation in $\mathbb{R}^{2}$. So now $Z \subseteq \mathbb{R}^{2}$ (i.e. $N=2$ ):

$$
\left.\begin{array}{c}
\frac{\partial x}{\partial t}-\xi \Delta x+(x \mathrm{D}) x=f(t, z, x) u-\mathrm{D} p, \quad \text { on } T \times Z, \\
\left.x\right|_{T \times \Gamma}=0, \quad x(0, z)=x(b, z), \quad \text { a.e. on } Z, \\
\|u(t, z)\| \leqslant r(x), \quad \text { a.e. on } T \times Z, \quad \xi>0 .
\end{array}\right\}
$$

Let $\mathcal{D}=\left\{x \in C_{0}^{\infty}\left(Z, \mathbb{R}^{2}\right): \operatorname{div} x=0\right\}$ and let $X=\overline{\mathcal{D}}$ (the closure taken in $H_{0}^{1}\left(Z, \mathbb{R}^{2}\right)$ ). We know that $X=\left\{x \in W_{0}^{1, p}\left(Z, \mathbb{R}^{2}\right): \operatorname{div} x=0\right\}$ (see Temam [20, p. 18]). Then, if $H=L^{2}\left(Z, \mathbb{R}^{2}\right),\left(X, H, X^{*}\right)$ is an evolution triple with compact embeddings.

Let $A \in \mathcal{L}\left(X, X^{*}\right)$ be defined by

$$
\langle A(x), y\rangle=\int_{Z} \sum_{i=1}^{2}\left(\mathrm{D} x_{i}, \mathrm{D} y_{i}\right)_{\mathbb{R}^{2}} \mathrm{~d} z
$$

This operator is clearly monotone (thus maximal monotone), coercive (i.e. $\langle A(x), x\rangle \geqslant$ $\left.c\|x\|^{2}\right)$.

Let

$$
b: X \times X \times X \rightarrow \mathbb{R}
$$

be the trilinear form defined by

$$
b(x, y, v)=\sum_{k, l=1}^{2} \int_{Z} x_{k}\left(D_{k} y_{l}\right) v_{l} \mathrm{~d} z
$$

Since $N=2$, Lemma 1.2 from Temam [20, p. 162], implies that $b$ is trilinear continuous, $b(x, y, y)=0$ and $b(x, y, v)=-b(x, v, y)$ for all $x, y, v \in X$. Then we can introduce $B: X \rightarrow X^{*}$ to be the nonlinear map defined by $\langle B(x), y\rangle=b(x, x, y)$. Lemma 3.2 from Temam $[20$, p. 289] implies that $B$ is sequentially weakly continuous.

We introduce the following hypotheses concerning $f(t, z, x)$.

$H(f)_{2}: f: T \times Z \times \mathbb{R}^{2} \rightarrow \mathbb{R}$ is measurable in $(t, z)$, Lipschitz continuous in $x \in \mathbb{R}^{2}$ and $|f(t, z, x)| \leqslant \beta(t, z)$ a.e. on $T \times Z$, for all $x \in \mathbb{R}^{2}$, where $\beta \in L^{\infty}(T \times Z)$.

Let $\hat{f}: T \times H \rightarrow H$ be defined by $\hat{f}(t, x)(\cdot)=f(t, \cdot, x(\cdot))$ and let $U: L^{2}\left(Z, \mathbb{R}^{2}\right) \rightarrow$ $L^{\infty}\left(Z, \mathbb{R}^{2}\right)$ be defined by $U(x)=\left\{u \in L^{\infty}\left(Z, \mathbb{R}^{2}\right):\|u(z)\| \leqslant r(x)\right.$, a.e. on $\left.Z\right\}$. Assume the following.

$H(r): r: L^{2}\left(Z, \mathbb{R}^{2}\right) \rightarrow \mathbb{R}_{+}$is Lipschitz continuous. 
Then, if $F(t, x)=\hat{f}(t, x) U(x)$, we can rewrite (4.3) in the following equivalent deparametrized (i.e. control-free) evolution inclusion form:

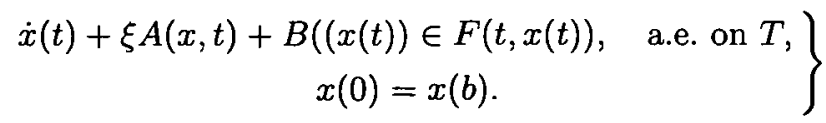

Using the properties of $B$, we can check that $\xi A+B$ is pseudomonotone and

$$
\langle(\xi A+B)(x), x\rangle \geqslant c\|x\|^{2} .
$$

Moreover, $F(t, \cdot)$ is $h$-Lipschitz. Thus, Theorem 3.1 can be applied. If $\eta: H \rightarrow \mathbb{R}$ is a continuous, coercive cost function and we consider the terminal cost problem

$$
\eta(x(b, \cdot)) \rightarrow \inf _{x \in S_{c}}=m
$$

then, using Theorem 3.1 we can have the following result concerning problem (4.5).

Theorem 4.3. If hypotheses $H(f)_{2}$ and $H(r)$ hold and $\varepsilon>0$, then we can find $x \in W_{2,2}(T)$ and $u \in \operatorname{ext} U(x)$ such that $\eta\left(x_{u}(b, \cdot)\right) \leqslant m+\varepsilon$; here, by $x_{u}$ we denote the state generated by the control $u$ (recall that $\operatorname{ext} F(t, x) \subseteq \hat{f}(t, x) \operatorname{ext} U(x)$ ).

Acknowledgements. The authors thank a knowledgeable referee for his/her remarks.

\section{References}

1. N. U. AHMED, An existence theorem for differential inclusions on Banach space, J. Appl. Math. Stoch. Analysis 5 (1992), 123-130.

2. G. ANICHINI AND P. ZECCA, Multivalued differential equations and control problems, Houston J. Math. 10 (1984), 307-313.

3. R. BECKER, Periodic solution of semilinear equations of evolution of compact type, $J$. Math. Analysis Appl. 82 (1981), 33-48.

4. F. BROWDER, Existence of periodic solutions for nonlinear equations of evolutions, Proc. Natn. Acad. Sci. USA 53 (1965), 1110-1113.

5. Z. DiNG AND A. KARTSATOS, Nonresonance problems for differential evolution equations in Banach spaces, Proc. Am. Math. Soc. 124 (1996), 2357-2365.

6. N. HIRANO, Existence of periodic solutions for nonlinear evolution equations in Hilbert spaces, Proc. Am. Math. Soc. 120 (1994), 185-192.

7. S. HU AND N. S. PAPAGEORGIOU, Handbook of multivalued analysis, vol. 1: Theory (Kluwer, Dordrecht, 1997).

8. S. HU AND N. S. PAPAGEORGIOU, On the existence of periodic solutions for a class of nonlinear evolution inclusions, Bolletino UMI 7-B (1993), 591-605.

9. S. HU AND N. S. PAPAGEORGIOU, On the existence of periodic solution for nonconvex valued differential inclusions in $\mathbb{R}^{N}$, Proc. Am. Math. Soc. 123 (1995), 3043-3050.

10. S. Hu, D. Kandilakis and N. S. Papageorgiou, Periodic solutions for nonconvex differential inclusions, Proc. Am. Math. Soc. 127 (1999), 89-94.

11. J.-L. LIONS, Quelques methodes de resolution des problemes aut limites nonlineaires (Dunod, Paris, 1969).

12. S. Migorski, On an existence result for nonlinear evolution inclusions, Proc. Edinb. Math. Soc. 39 (1996), 133-141. 
13. N. S. PAPAgeorgiou, On the set of solutions of a class of nonlinear evolution inclusions, Kodai Math. J. 15 (1992), 387-402.

14. N. S. PAPAgEORgIOU, A continuous version of the relaxation theorem for nonlinear evolution inclusions, Houston J. Math. 20 (1994), 685-704.

15. N. S. PAPAgeorgiou, Boundary value problems and periodic solutions for semilinear evolution inclusions, Commun. Math. Univ. Carol. 35 (1994), 325-326.

16. N. S. PAPAGEORgIOU, On the existence of solutions for nonlinear parabolic problems with nonmonotone discontinuities, J. Math. Analysis Appl. 205 (1997), 434-453.

17. N. S. Papageorgiou, F. PAPAlini AND F. RenZACCI, Existence of solutions and periodic solutions for nonlinear evolution inclusions, Rend. Circolo Mat. Palermo 48 (1999), 341364 .

18. N. S. Papageorgiou And N. Shahzad, Properties of the solution set of nonlinear evolution inclusions, Appl. Math. Optim. 36 (1997), 1-20.

19. J. Pruss, Periodic solutions of semilinear evolution equations, Nonlinear Analysis 3 (1979), 601-612.

20. R. TEMAM, Navier-Stokes equations: theory and numerical analysis, 3rd edn (North Holland, Amsterdam, 1984).

21. J. VRABIE, Periodic solutions for nonlinear evolution equations in a Banach space, Proc. Am. Math. Soc. 109 (1990), 653-661.

22. E. ZEIDLER, Nonlinear functional analysis and its applications, vol. II (Springer, New York, 1990). 